\title{
RASMUNSSEN ENCEPHALITIS IN YOUNG ADULT: A CASE REPORT
}

\author{
Nicoleta Tohanean ${ }^{1}$, Oana Vanta ${ }^{1}$, Alexandru Centea ${ }^{1}$, Teodora Alexescu², \\ Lacramioara Perju-Dumbrava ${ }^{1}$ \\ ${ }^{1}$ Neurology Department, \\ "Iuliu Hatieganu" University of Medicine and Pharmacy Cluj-Napoca, Romania \\ ${ }^{2} 5^{\text {th }}$ Internal Medicine Department, $4^{\text {th }}$ Medical Clinic, \\ "Iuliu Hatieganu” University of Medicine and Pharmacy, Cluj-Napoca, Romania
}

\begin{tabular}{|l} 
ABSTRACT \\
Rasmunssen encephalitis is a rare disease characterized by focal cortical inflammation with progressive extension \\
and diffusion. Clinical features are continuous partial epilepsy non responsive to usual anti-epileptic therapy and \\
unilateral motor deficit, corresponding with the IRM imaging to cerebral hemiatrophy. The disease mainly affects \\
children but there are some acknowledged cases described in adolescent and adult life. \\
This report aims to present a clinical case of a young female with seven years personal history of generalized \\
epilepsy. Having a clinical and paraclinical evolution a suspicion of chronic focal Rasmunssen encephalitis took \\
shape. During admission she suffered of weakness and involuntary movements in her left side, which gradually \\
worsened in the last month and memory impairment also. Routine laboratory blood evaluation, inflammatory and \\
infectious tests were within the normal range. Magnetic resonance imaging of the brain reveals T2 atrophy of the \\
right cerebral hemisphere with a low displacement of median structures to the left and an abnormal hyper inten- \\
sity signal on T2-weighted, along with FLAIR images. Electroencephalography showed a profoundly altered, \\
slowed, asymmetrically with paroxysmal unilateral right slow activity record with high amplitude. \\
Based on clinical criteria, MRI imaging and EEG we supported the diagnosis of Rassmunsen encephalitis. \\
Keywords: Rasmunssen, continuous partial epilepsy, antibodies anti-GluR3
\end{tabular}

Keywords: Rasmunssen, continuous partial epilepsy, antibodies anti-GluR3

\section{INTRODUCTION}

The entity of Rasmunssen Encephalitis (RE) was described in 1958 by Theodore Rasmussen who reported three subjects with focal seizures in the context of autoimmune localized encephalitis $(1,2) . \mathrm{RE}$ is a rare and heterogenic condition that was mainly found in children, showing associated motor deficits or other cortical signs (1-3).

There are many terms to describe this condition: chronic localized/focal encephalitis; epilepsy associated with hemiplegia and intellectual disabilities but the most used is Rasmussen syndrome or Rasmunssen Encephalitis (RE) (1).

Clinical characteristics are represented by drugresistant focal epilepsy, progressive cognitive deterioration and hemiplegia, caused by unilateral in- flammation of the cerebral cortex (4). This is a severe encephalopathy of unknown etiology characterized by inflammation and focal and extensive necrosis of the cerebral cortex. As noticed, the most affected are children between 2 and 10 years in $85 \%$ of cases (5) but there are a few exceptional cases reported in adolescence and young adults (4).

The exact etiology of the RE remains unknown. Several hypotheses have been proposed: viral, involvement of circulating autoantibodies, but most probable it seems to be the autoimmune hypothesis involving both humoral immunity, as well as T cell mediated cytotoxicity $(1,4)$. Antibodies anti-GluR3 (against subunit 3 of the ionotropic glutamate receptor) seem to play an important role but they are not specific for RE because they are not present in 
all RE patients, and they might be found in other epilepsy types $(1,4)$. Cytotoxic $\mathrm{T}$ lymphocytes CD8+ are also involved, attacking some autoantigens expressed by neurons and astrocytes or some foreign antigens from pathogen-infected neurons $(4,6)$ and are responsible for cellular destruction leading to massive necrosis of one cerebral hemisphere (7). Another morphopathological aspect recently described in ER is microglial activations and this seems to increase brain excitability (4).

Positive diagnosis of RE is based on clinical criteria, MRI imaging and EEG findings (1).

EEG is useful in early diagnosis of RE and can highlight unihemispheric changes with polymorphic delta waves, mainly in a temporal and central locationand multifocal ictal discharges $(1,8)$.

MRI examination can describe unilateral enlargement of the inner and outer CSF compartments, with increased cortical or subcortical T2 and FLAIR signal and atrophy of the head of the caudate nucleus on the same side. CT scan in early cases, may reveal in some patients transiently focal cortical swelling $(8,9)$.

CSF analysis helps to eliminate other diseases, especially infections of the central nervous system. Measurements of GluR3 antibodies in serum and CSF are used but are not specific and do not differentiate between RE and other types of epilepsy (1).

\section{CASE PRESENTATION}

We report a case of a 25 year old female patient that was admitted in our Department in November 2014 for two to three generalized seizures/day accompanied by weakness and involuntary movements in her left side, symptoms that developed gradually in the last month. She also described memory impairment, dyscalculia, accentuated sleepiness and impaired concentration.

From the age of 18, the patient was known to have generalized seizures and was diagnosed with idiopathic epilepsy and underwent treatment with valproic acid $1,000 \mathrm{mg} /$ day. Nor the patient or her family had any notion of birth suffering and they described a normal psychomotor development.

During neurological examination we observed a patient with abnormal gait, postural instability in need of a wide base and positive Romberg sign, left central facial palsy; left flaccid hemiparesis with MS-3/5 in the left upper limb and MS-2/5 in the left lower limb with increased osteotendinous reflexes and the presence of Babinski sign; impaired sensory perception in her left side - tactile hypoesthesia, hypalgesia, thermohypoestesia and reduced vibra- tory sense; speech impairment - explosive speech and bradylalia, bradyphrenia, dyscalculia, memory impairment.

Blood analysis did not reveal any inflammatory response - ESP, CRP and WBC were in normal range. Infectious causes like Herpes Simplex virus 1 and 2, HIV, Varicella-Zoster Virus, Epstein-Barr Virus, Borrelia Burgdorferi, Toxoplasma Gondii were also excluded.

A lumbar tap was performed to rule out CNS infections. CSF analysis revealed normal cell counts and protein levels. The CSF IgG index was also normal.

The electroencephalogram (EEG) showed slowing of the background activity rhythm along with an asymmetry: right side of paroxysmal abnormalities with slow wave and spikes-slow waves complex, singly or in group, mainly in temporal region.

The CT scan revealed important cerebral oedema of the right hemisphere with loss of white - gray matter differentiation, effaced sulci and right lateral ventricle with $3 \mathrm{~mm}$ deviation to the left of the midline structures. No contrast enhancement of brain parenchyma was observed.

The brain MRI examination revealed in axial T2-weighted MR image atrophy of the right cerebral hemisphere. This was evidenced by the dilatation of the ipsilateral lateral ventricle, right Sylvian fissure, subarachnoid spaces and widening of the cortical sulci, most marked at the temporal lobe. There was a low displacement of median structures to the left by about $3 \mathrm{~mm}$. The frontal horn, the body, the temporal and occipital horn of the ipsilateral ventricle showed minimum deformation mass effect.

Gray matter showed abnormal hyper intensity signal on T2-weighted and fluid-attenuated inversion recovery (FLAIR) images. This cortical and subcortical swelling with hyper intense T2/FLAIR signal were also seen in DWI and ADC sequences. The insular, frontal and temporal regions were the most affected areas, and minimal in parietal and occipital regions.

This unilateral cortical atrophy with ex-vacuo ventricular dilatation, with hyper intense $\mathrm{T} 2 \mathrm{~W}$ signal areas in the right hemisphere, with no modifications on the left hemisphere, with restricted diffusion DWI/ADC in altered signal areas raised the suspicion of chronic focal encephalitis - Rasmunssen encephalitis.

The patient received cerebral depletion therapy and a combination of antiepileptic drugs- I.V Levetiracetam $1,000 \mathrm{mg} /$ day and valproic acid $1,000 \mathrm{mg} /$ day but the treatment was ineffective. The patient 
presented recurrent left versive focal motor seizures occurring every few minutes that developed into secondary generalized seizures with loss of consciousness and prolonged postictal somnolence.

Given the patient's symptoms, the EEG findings, the MRI imaging features and the lack of a possible infectious agent we supported the idea of an autoimmune response and, thus diagnosed the patient with Rasmussen's Encephalitis. We initiated pulse corticosteroid therapy with methylprednisolone (6 grams in 7 days), and concequently we noticed a significant improvement in the frequency of partial motor seizures - the patient presented only 6-7 partial left motor seizures/day without generalization.

The patient was discharged with indication for oral corticosteroid therapy with methylprednisolone in slow decreasing doses and a combination of antiepileptic drugs - levetiracetam $1 \mathrm{~g} /$ day and valproic acid $1 \mathrm{~g} /$ day.

On the three months assessment we observed a significant clinical improvement - that is the patient was seizure free with a residual spastic hemiparesis. The EEG findings were consistent with the clinic - more decelerated background activity but without paroxysmal discharges.

\section{DISCUSSION}

$\mathrm{RE}$ is a rare entity that mainly affects children aged between 2 and 10 years in $80 \%$ of cases. There are few cases described in adolescents and young adults $(5,10,11)$.

The clinical presentation may be represented by three different forms of evolution that correlates with the imaging changes and the pathological lesions. From the point of view of clinical evolution, Bien describes in his studies three stages of disease $(1,3,7)$.

- prodromal phase (median duration of 7.1 months) characterized by the occurrence of mild epilepsy with seizures relatively rare, exceptionally associated with hemiparesis.

- acute phase (median duration of 8 months), during which the crises are frequent (partial motor crises) but often could appear continuous partial epilepsy. In this stage, other neurological signs occur: progressive hemiparesis, hemianopia, cognitive deterioration and aphasia.

- residual phase characterized by persistent neurological deficits - hemiparesis with transient improvement of epilepsy, still difficult to control $(1,3,7)$.
Our patient was diagnosed during the acute phase of the disease.

In order to establish the diagnosis of RE, there are no available specific exams (1). Antibodies antiGluR3 antibodies in serum (and CSF alike) are positive but nonspecific and they do not discriminate between RE and others forms of resistant epilepsy (1).

There are some peculiarities of clinical presentation, evolution and response to treatment in adults versus children.

Literature tell us that the late-onset form of the disease is characterized by a mean age at onset of 24 years and female predominance. Neurological deficits occur less often as in children, $75 \%$ in adults compared to $100 \%$ in children. The most common neurological impairment is due to bilateral occipital lobe insult with the early onset of occipital lobe seizures $(1,10-11)$. The evolution in adult life is slower and less severe as that described in children. The average duration of neurologic deficits is 11.6 years $(1,10-11)$. Electrophysiological, neuroimaging and histopathological findings are the same as those described in children $(1,2)$.

Clinical differential diagnostic has been done with other neurological entities that are unilateral and manifest along with continuous partial epilepsy, drug unmanageable (1).

Imagistic differential diagnostic for hemimegaloencephalia is based on the craniocerebral symmetry which is present in this case. Other differential diagnoses can be made with other syndromes that evolve with epilepsy and cerebral hemiatrophy: like Dyke-Davidoff-Masson syndrome and ParryRomberg syndrome, but there are other clinical features as mental retardation and facial hemiatrophy $(10,11)$.

The goal of the treatment is to relieve the epileptic syndrome and to stop the progression of neurological deficits and thus the neuronal loss (1).

The treatment is mainly medical in adults, consisting of corticosteroid medication, immunosuppressants or immunoglobulins. In adult onset RE, there are some studies using intravenous immunoglobulins, having good results (12). Seizures are very difficult to control and the literature doesn't talk about any antiepilepticregimen in mono or polytherapy, as being more effective as in other regimens (1).

In the case of children, we have the possibility of hemispherectomy, which improves epilepsy and reduces overall deterioration, but this form of treatment is not possible in adults due to functional reasons (10). 


\section{CONCLUSIONS}

It is important to remember that $\mathrm{RE}$ is a severe form of drug-resistant epilepsy that can occur in young adults and there are some distinctive features of evolution compared to those described in children. Early diagnosis of RE enables rapid establishment of medical treatment, limiting the progression of neurological deficits and intellectual deterioration.
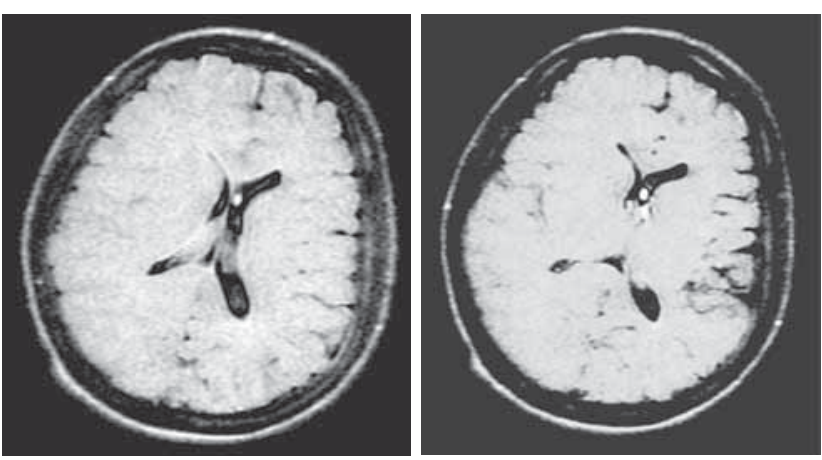

FIGURE 1. FLAIR IRM image and FIGURE 2. T1 IRM image: unilateral frontotemporal right cortical atrophy, with a minimum deviation to the left of the midline structures. We see no modifications on the left hemisphere
Controlled multicenter studies seem essential to define a consensual medical treatment strategy in forms with late onset.

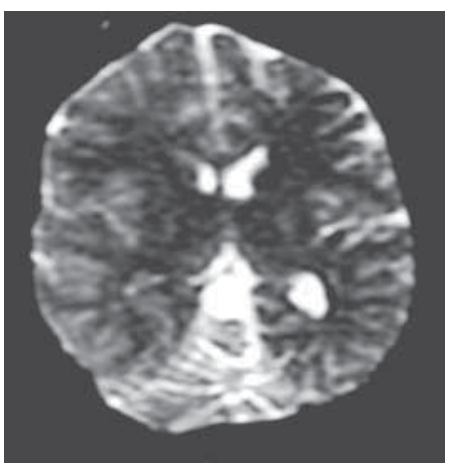

FIGURE 3. Diffusion IRM image: cortical and sub cortical swelling with slight hyper intense signal

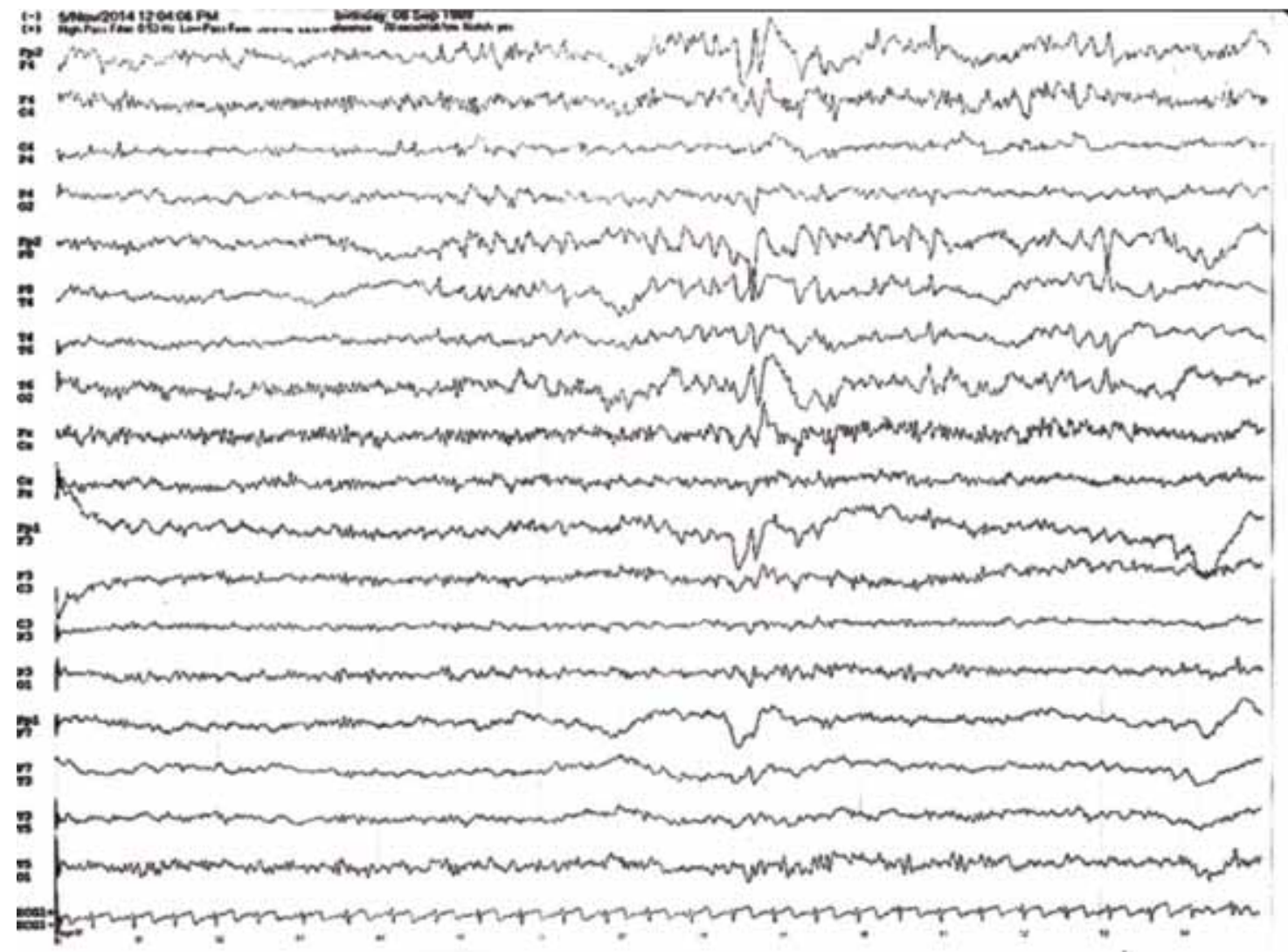

FIGURE4. Routine 18-channel digital EEG: slowed, asymmetrically record with paroxysmal, unilateral, mainly temporal, right slow activity with high amplitude-delta slow wave and spikes-slow waves complex, isolated or in short paroxysms. 


\section{REFERENCES}

1. Bien C.G., Granata T., Antozzi C., et al. Pathogenesis, diagnosis and treatment of Rasmussen encephalitis: a European consensus statement. Brain. 2005; 128: 454-71.

2. Owolabi M. Rasmussen's Encephalitis: An Overview. In: Dr. Daisuke Hayasaka (Ed.). Pathogenesis of Encephalitis, InTech, 2011: 235-254. Available from: http://www.intechopen.com/books/ pathogenesis-of-encephalitis/rasmussen-s-encephalitis-an-overview

3. Oguni H., Andermann F., Rasmunssen T.B. The naturalhistory of the syndrome of chronicencephalitis and epilepsy:astudy of the MNI series of forty-eight cases. In: F Andermann (ends).

Chronicencephalitis and epilepsy: Rasmussen's syndrome. Boston, Butterworth-Heinemann, 1991: 7-35

4. Varadkar S., Bien C.G., Kruse C.A., et al. Rasmussen's encephalitis: clinical features, pathobiology, and treatment advances. Lancet Neurol. 2014; 13(2): 195-205

5. Freeman J.M. Rasmussen's syndrome: progressive autoimmune multi-focal encephalopathy. Pediatr Neurol. 2005; 32: 295-299.

6. Bahi-Buisson N., Nabbout R., Plouin P., et al. Avancéesactuelles sur les concepts pathogéniques et thérapeutiques de l'encéphalite de Rasmussen. Rev Neurol. 2005; 161: 395-405
7. Bien C.G., Urbach H., Deckert M., et al. Diagnosis and staging of Rasmussen'sencephalitis by serial MRI and histopathology. Neurology. 2002; 58: 250-257.

8. Granata T., Gobbi G., Spreafico R., et al. Rasmussen's encephalitis: early characteristics allow diagnosis. Neurology. 2003; 60: 422-5.

9. Chiapparini L., Granata T., Farina L., et al. Diagnostic imaging in 13 cases of Rasmussen's encephalitis: can early MRI suggest the diagnosis? Neuroradiology. 2003; 45: 171-83.

10. Jaillon-Riviere V., Dupont S., Bertran F., et al. Late onset Rasmussen's syndrome: clinical and therapeutic characteristics. Rev Neurol. 2007; 163:573-580

11. El Assasse L., En-Nafaa l., Amraoui F., et al. Encéphalite de Rasmussen chez l'adulte: à propos d'un cas. Journal de radiologie. 2011; 92: 942-944

12. Granata T. Rasmussen's syndrome. Neurological Sciences. 2003; 24: 239-243 